\title{
Programa de locomoción y uso de transporte público para estudiantes con discapacidad intelectual: reporte de caso
}

\author{
Locomotion and use of public transportation program \\ for students with intellectual disability: case report
}

\author{
Pamela Soledad Torres Cáceres ${ }^{1}$
}

Recibido: 5 de enero 2018 • Enviado para modificación: 10 de junio 2018 • Aceptado: 15 de junio 2018 Torres-Cáceres, P. (2018). Programa de locomoción y uso de transporte público para estudiantes con discapacidad intelectual: reporte de caso. Revista Ocupación Humana, 18(1), 34-49.

\section{RESUMEN}

Se reporta el caso de dos estudiantes jóvenes con discapacidad intelectual, con el fin de evaluar el efecto de su participación en un programa de locomoción y uso del transporte público, realizado por el equipo de Terapia Ocupacional en el contexto de una escuela especial en Chile. Se propone una herramienta de evaluación e intervención centrada en el usuario, la escuela y la familia. Para dicha evaluación se utilizó el Modelo Kawa, buscando identificar dificultades y fortalezas que influyen en las elecciones ocupacionales durante el programa. La Evaluación de las Habilidades de Comunicación e Interacción Social y el Cuestionario Volitivo del Modelo de Ocupación Humana se usaron para evaluar la capacidad de desempeño y la volición en el inicio y la finalización del programa. Los dos estudiantes mostraron el fortalecimiento de los procesos volitivos y el aumento de habilidades de comunicación e interacción social, tanto en el contexto de la sala de clases como en las sesiones del programa. El programa puede tener implicaciones socioculturales, ya que enfatiza en la autonomía como un derecho que aporta a la libre elección ocupacional, dotando de dignidad a las personas, fortaleciendo su identidad y el descubrimiento de habilidades y capacidades no exploradas.

\section{PALABRAS CLAVE}

locomoción, Terapia Ocupacional, discapacidad intelectual, desarrollo de programa, educación especial, jóvenes

\section{ABSTRACT}

This article presents the case report of two young students with intellectual disability, with the purpose of evaluating the effect of their participation in a program for mobility and use of public transportation, created by the occupational therapy team at a special needs school

\footnotetext{
1 Terapeuta ocupacional. Docente campo clínico, Universidad San Sebastián, Sede Concepción. Concepción, Chile. pame.torres.c@gmail.com iD https://orcid.org/0000-0003-4482-4629
} 
in Chile. It proposes a tool for evaluation and intervention focused in the user, the school and the family. For this evaluation, the Kawa Model was used seeking to identify difficulties and strengths that influence the occupational choices during the program. The Assessment of Communication and Interaction Skills, and the Volitional Questionnaire were used to evaluate the performance and volition at the beginning and in the end of the program. The two students showed an enhancement of their volitional processes and the increase in their communication and social interaction skills, both in the classroom context, and in the occupational therapy sessions throughout the program. This program may have socio-cultural implications, since it emphasizes in autonomy as a right that contributes to free occupational choices, which provide dignity to people, enhancing their personal identity and the discovery of skills not explored previously.

\section{KEY WORDS}

locomotion, Occupational Therapy, intellectual disability, program development, special education, youth

\section{Introducción}

La Federación Mundial de Terapeutas Ocupacionales -WFOT(2012) define la Terapia Ocupacional como la profesión de salud centrada en el cliente que se preocupa por promover la salud y el bienestar a través de la ocupación. Su objetivo principal es permitir que las personas participen en las ocupaciones que desean, necesitan o se espera que realicen, aumentando sus capacidades o modificando la ocupación o el ambiente. En efecto, el foco de la profesión es la participación de las personas en la sociedad mediante actividades significativas. Si pensáramos en el transporte público, determinaríamos que este es esencial para que las personas accedan a la educación, el empleo, los servicios de salud, a eventos sociales y actividades de ocio. Sin embargo, la falta de medios accesibles para realizar viajes independientes crea exclusión social para muchas personas con discapacidad (Alsnih \& Hensher, 2003; Heiser, 1995; Social Exclusion Unit, citado en
Haveman, Tillmann, Stöppler, Kvas \& Monninge, 2013).

Se entiende por discapacidad intelectual aquella que se deriva de limitaciones significativas en el funcionamiento intelectual y el comportamiento adaptativo, que se expresan en habilidades adaptativas conceptuales, sociales y prácticas (Schalock et al., 2010). Aquellos jóvenes que presentan discapacidad intelectual ven limitada su independencia al tratar de usar los medios de transporte sin la compañía de un adulto, sin embargo, muchos de ellos anhelan tener una mayor autonomía para realizar distintas actividades y contar con una mejor preparación para lograrlo (Haveman et al., 2013).

Como antecedente, en Alemania se realizó el "Estudio de intervención del transporte público de Nordhorn, Alemania" (Haveman et al., 2013), que buscaba aumentar el uso independiente del transporte público por parte de estudiantes con discapacidad intelectual. 
Con este proyecto, a través de intervenciones en el entorno social y físico, y con un seguimiento de tres años, se lograron mejoras estadísticamente significativas en las habilidades para usar el transporte público y en el comportamiento de orientación.

De otro lado, se han creado evaluaciones como el Listado de Evaluación de Movilidad -MAS², que mide las habilidades básicas de tráfico en estudiantes con discapacidad intelectual entre los 7 y los 18 años de edad, abarcando ocho dominios: percepción visual, percepción auditiva, reacción, memoria, atención, habilidades motrices, comunicación y habilidades sociales (Kvas, Stöppler, Haveman \& Tillmann, 2013).

Sin embargo, ninguno de los estudios mencionados ha sido realizado por terapeutas ocupacionales, tampoco enfatizan en aspectos personales como la percepción de los participantes, sus temores, la credibilidad en sus habilidades para lograr el uso del transporte público de manera independiente, las transformaciones personales a nivel de la confianza en su propia autonomía el fortalecimiento de su identidad o la competencia a través de la libertad de exploración del medio.

\section{Descripción del programa}

El Programa de Locomoción y Uso de Transporte Público tiene por objetivo aportar a jóvenes estudiantes con discapacidad intelectual en el desarrollo de su capacidad de moverse libremente en la ciudad, sin la compañía de un adulto, propiciando el desarrollo de habilidades de comunicación e interacción social, y el fortalecimiento de los componentes volitivos. En este sentido, la escuela cumple un papel importante como agente de apoyo en los proyectos de vida, teniendo como fin acortar la brecha entre la realidad y la inclusión social (Rusch, Hughes, Agran, Martin \& Johnson, 2009).

El programa fue desarrollado en una escuela especial de la provincia de Concepción, Chile. Esta institución cuenta con un equipo profesional integrado por: psicólogo, fonoaudiólogo, asistente social y educadoras diferenciales. Los estudiantes que asisten a la escuela, de entre 2 y 25 años de edad, presentan discapacidad intelectual y en algunos casos otros diagnósticos motores o sensoriales asociados. El servicio de Terapia Ocupacional se incorpora a la escuela en el año 2015, mediante convenio con la Universidad San Sebastián; su principal objetivo es aportar a la autonomía e independencia de los estudiantes mediante talleres e intervenciones individuales.

El grupo de Terapia Ocupacional de la escuela creó el Programa en el año 2017, desde su enfoque profesional y tomando como referentes teóricos el Modelo Kawa (Río) y el Modelo de Ocupación Humana, además de considerar los estudios mencionados previamente, el "Programa de desarrollo de objetivos de aprendizaje para los niveles de $1^{\circ}$ a $6^{\circ}$ básico en el área de educación vial" (Conaset \& Ministerio de Transporte y Telecomunicaciones del Gobierno de Chile, 2017) y el documento "Transición para la vida adulta" (Quintana \& Barrera, 2013).

${ }^{2}$ La sigla MAS corresponde a las iniciales en inglés de Mobility Assessment Schedule. 
Para conformar las unidades del programa se trabajó con metodología activa y participativa junto a educadoras diferenciales ${ }^{3}$, enfatizando en las características de los jóvenes, Ilegando a incorporar cinco unidades temáticas (tabla 1).
El Modelo Kawa permite al terapeuta ocupacional acceder a una nueva forma de comprender holísticamente la vida $y$, por ende, al individuo, no solo su condición de salud o el momento de transición que requiere del apoyo o servicio profesional. Los conceptos de

Tabla 1. Contenidos del Programa de Locomoción y Uso de Transporte Público

\begin{tabular}{|c|c|c|c|c|c|c|}
\hline & Contenidos & Temporalidad & $\begin{array}{l}\text { Gufas } \\
\text { Hogar }\end{array}$ & $\begin{array}{c}\text { Apoyo } \\
\text { Terapia } \\
\text { Ocupacional }\end{array}$ & $\begin{array}{l}\text { Apoyo } \\
\text { Educación } \\
\text { Diferencial }\end{array}$ & $\begin{array}{l}\text { Apoyo } \\
\text { Familla }\end{array}$ \\
\hline $\begin{array}{c}\text { Unidad } \\
\text { I }\end{array}$ & $\begin{array}{l}\text { Recolección de información en } \\
\text { visitas domiciliarias registradas } \\
\text { en notas de campo. }\end{array}$ & Marzo & $x$ & $x$ & $x$ & $x$ \\
\hline $\begin{array}{l}\text { Unidad } \\
\text { II }\end{array}$ & $\begin{array}{l}\text { Aplicación de modelo Kawa. } \\
\text { Participación en diálogos } \\
\text { reflexivos con los padres, } \\
\text { educadoras y las personas } \\
\text { participantes del programa. } \\
\text { Reconocimiento espacial de } \\
\text { lugares aledarios. Determinar } \\
\text { puntos clave de toma de } \\
\text { transporte público, cercanos a la } \\
\text { escuela. Utilizar leyes del } \\
\text { tránsito, identificar los recorridos } \\
\text { de líneas especificas de buses } \\
\text { que transitan por el sector. }\end{array}$ & Nbril-Mayo & $x$ & $\mathbf{x}$ & $\mathrm{x}$ & $x$ \\
\hline $\begin{array}{l}\text { Unidad } \\
\text { III }\end{array}$ & $\begin{array}{l}\text { Incorporar hábitos y normas } \\
\text { involucradas con el uso de } \\
\text { medios de transporte, como: } \\
\text { subirse al bus, saludar, pagar el } \\
\text { pasaje, recibir y guardar el } \\
\text { boleto, sentarse, ceder el asiento } \\
\text { a las mujeres embarazadas, } \\
\text { ancianos y personas con } \\
\text { dificultades físico/motoras, hablar } \\
\text { en voz baja, mantener la } \\
\text { limpieza en el bus, pedir permiso } \\
\text { al momento de bajar del bus en } \\
\text { caso de requerirlo, entre otras. }\end{array}$ & Junio & $x$ & $x$ & & $x$ \\
\hline $\begin{array}{l}\text { Uniderd } \\
\text { IV }\end{array}$ & $\begin{array}{l}\text { Desarrollar las capacidades } \\
\text { asertivas durante la utilización } \\
\text { del transporte público. Pasos por } \\
\text { seguir en caso de accidente, } \\
\text { volcamiento, incendio y otras } \\
\text { situaciones cotidianas como: } \\
\text { bajarse en un lugar desconocido } \\
\text { o ir retrasado a un encuentro } \\
\text { social. }\end{array}$ & Julio & $x$ & $x$ & & $x$ \\
\hline $\begin{array}{l}\text { Unidad } \\
V\end{array}$ & $\begin{array}{l}\text { Locomoción y uso del transporte } \\
\text { público en la comunidad, con } \\
\text { base en los intereses y } \\
\text { necesidades, con apoyo } \\
\text { permanente de la familia en la } \\
\text { supervisión y posterior } \\
\text { independencia. }\end{array}$ & $\begin{array}{l}\text { Julio- } \\
\text { Noviembre }\end{array}$ & & $x$ & & $x$ \\
\hline
\end{tabular}

Fuente: Elaboración propia a partir del desarrollo de objetivos de aprendizaje para los niveles de $1^{\circ}$ a $6^{\circ}$ básico en el área de educación vial.

${ }^{3}$ La denominación educador(a) diferencial, utilizada en Chile, es equivalente a la de educador(a) especial, empleada en otros países de habla hispana. 
este Modelo son idealmente significativos, reconocidos y valorados por la persona (Castro de Jong et al., 2010).

En el marco del Modelo Kawa es posible analizar, desde la visión particular de los estudiantes con discapacidad intelectual, las dificultades y fortalezas asociadas a la locomoción y el uso del transporte público. De acuerdo a sus historias de vida, pueden relacionarse obstáculos, factores personales y ambientales que influyen en la capacidad de realizar elecciones ocupacionales de forma libre y autónoma, según las oportunidades del medio.

Teniendo en cuenta lo anterior, la metáfora del río que propone este modelo se transforma en una herramienta de diagnóstico inicial, aplicada individualmente. El terapeuta realiza una reseña de los elementos de la metáfora y su asociación con la locomoción y el uso del transporte público, dando libertad a los estudiantes para expresar cada elemento en una hoja en blanco, representando aquellos aspectos que consideran relevantes.

Los elementos centrales de la metáfora del río, asociados a la locomoción y uso de transporte público, son:

Iwa (rocas): se refiere a las circunstancias que afectan el flujo de la vida y que son percibidas por la persona como problemáticas y difíciles de quitar (Iwama \& Simó, 2008). Ejemplo: "no me dejan salir sola, tengo miedo a cruzar la calle".

Ryuboku (madera flotante): serían las cualidades personales y los recursos percibidos por la persona, tales como amigos, valores, carácter, personalidad (Iwa- ma \& Simó, 2008). Ejemplo: "me gusta hablar con personas desconocidas, soy desobediente, creo en mis capacidades para usar el transporte público".

Kawa no soku heki (paredes)/ Kawa no zoko (lecho del río): representan el entorno del sujeto (Iwama \& Simó, 2008). Ejemplo: "vivo en un lugar peligroso, mi familia no quiere dejarme usar el transporte público".

De otro lado, el Modelo de Ocupación Humana (Kielhofner \& Forsyth, 2011) provee un marco en el cual cobran sentido aspectos volitivos como la sensación de reconocer y creer en las propias capacidades al realizar actividades de locomoción y uso de transporte público, los intereses personales, el fomento de la responsabilidad en la locomoción y el desarrollo de la iniciativa para usar el transporte público. Del mismo modo, la generación de hábitos y rutinas en conjunto con la familia y la escuela, los cuales permitan incorporar la locomoción y el uso de transporte como parte de las actividades cotidianas. Por último, la capacidad de desempeño, considerando el cuerpo vivido como componente subjetivo, y las capacidades y habilidades sociales, motoras y de procesamiento de los estudiantes, como componente objetivo. Adicionalmente, se considera al ambiente social y físico como parte fundamental en el logro de la locomoción y el uso del transporte público de manera independiente (figura 1).

El Modelo de Ocupación Humana además suministra herramientas que se utilizan en el Programa, puesto que permiten evaluar la evolución de las habilidades de comunicación e interacción social, así como los componentes 
Figura 1. Elementos del Modelo de Ocupación Humana aplicados al Programa de Locomoción y Uso del Transporte Público

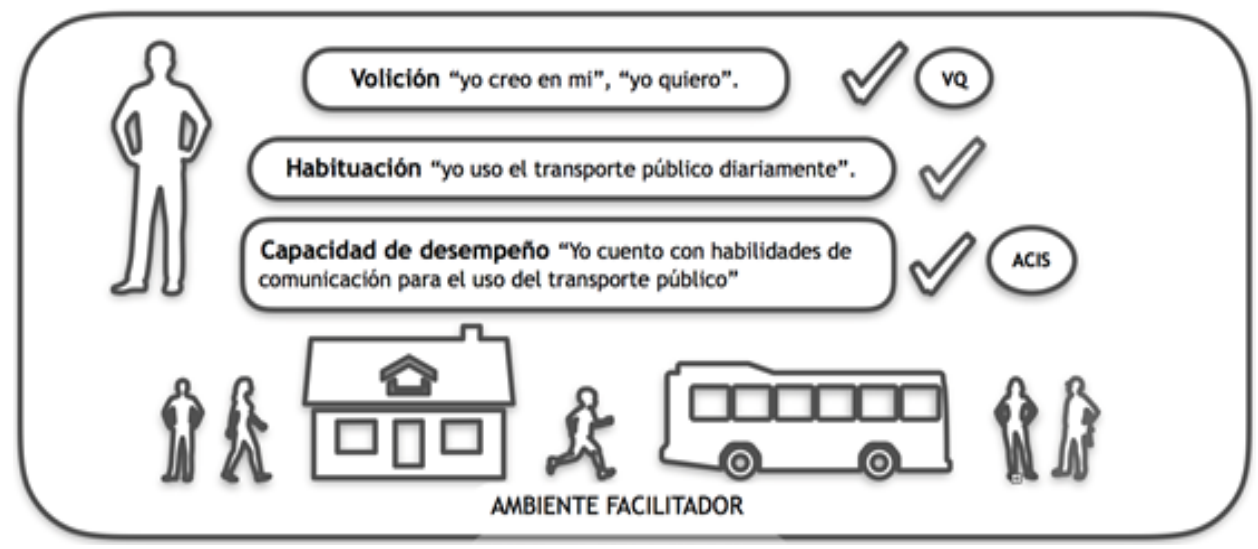

Fuente: Elaboración propia

volitivos, estas son, la Evaluación de las Habilidades de Comunicación e Interacción Social-ACIS y el Cuestionario Volitivo -VQ4.

El ACIS evalúa, a través de la observación, 20 ítems distribuidos en tres dominios: dominio físico (6 ítems), dominio de intercambio de información (9 ítems) y dominio de relaciones (5 ítems). El puntaje a obtener por cada indicador es de 1 a 4 , siendo 1 déficit en el desempeño, 2 desempeño inefectivo, 3 desempeño cuestionable y 4 desempeño competente. El puntaje total de la evaluación son 80 puntos. El Manual del Usuario de la Evaluación de las Habilidades de Comunicación e Interacción (Forsyth, 1998) reporta tres estudios de viabilidad del ACIS, realizados por Simon (1989), Salamy (1993) y Forsyth (1996), este último mostró resultados en 19 ítems que se unen para formar un contenido unidimensional, lo cual se traduce en una validez interna de la herramienta.

Entre tanto, el Cuestionario Volitivo -VQ cuenta con 14 ítems que se puntúan de 1 a 4, describiendo el grado de espontaneidad demostrado por la persona: 1 , pasivo; 2 , dudoso; 3 , involucrado, y 4, espontáneo. El puntaje total del cuestionario es de 56 puntos. Este cuestionario se basa en hallazgos de investigación y en la práctica clínica, cuenta con un criterio de puntaje refinado, así como con un sistema eficiente para registrar información sobre el ambiente (De las Heras, Geist, Kielhofner, \& Li, 1998).

Adicionalmente, el grupo de terapeutas ocupacionales y educadoras diferenciales elaboró una pauta de evaluación con criterios para cada unidad,

\footnotetext{
${ }^{4}$ Las siglas ACIS y VQ corresponden a las iniciales de estos instrumentos en inglés: Assessment of Communication and Interaction Skills - ACIS y Volitional Questionnary-VQ.
} 
basados en el artículo 8 del Decreto 87 de 1990, a través del cual se aprueban planes y programas de estudio para personas con discapacidad intelectual en Chile. Así, el progreso de los alumnos se determina a través de la valoración funcional de los aprendizajes, registrada en las asignaturas y actividades, de acuerdo a la siguiente escala: objetivo logrado (L), objetivo en desarrollo (OD) y objetivo no logrado (NL) (Gobierno de Chile, Decreto 87, 1990) (tabla 2).

La implementación del programa inicia con una visita domiciliaria
(Unidad I) registrada en notas de campo, identificando temores familiares relacionados con la locomoción y el uso del transporte público. Posteriormente, en la Unidad II se analizan los constructos del modelo Kawa y se aplican en el contexto de la escuela. Posteriormente, se presentan los resultados a las familias y se generan estrategias que aporten al programa, como el apoyo permanente a nivel socio emocional y la generación de guías prácticas que refuercen los contenidos tratados. Se aplica la Evaluación de Habilidades de Comunicación e Inte-

Tabla 2. Pauta de evaluación del Programa de Locomoción y Uso del Transporte Público

\begin{tabular}{|c|c|c|c|c|c|}
\hline Unidades & & Indicadores & OL & OD & NL \\
\hline \multirow{4}{*}{$\begin{array}{c}\text { Unidad I: } \\
\text { Percepción de las } \\
\text { familias sobre la } \\
\text { locomoción y el uso del } \\
\text { transporte público } \\
\text { independiente }\end{array}$} & 1 & Reconoce la importancia de la locomoción independiente. & & & \\
\hline & 2 & $\begin{array}{l}\text { Percibe las barreras contextuales que dificultan la locomoción } \\
\text { independiente de su hijo/a. }\end{array}$ & & & \\
\hline & 3 & $\begin{array}{l}\text { Desarrolla actitudes que favorecen la confianza en sus hijos para } \\
\text { la toma de desafíos. }\end{array}$ & & & \\
\hline & 4 & Entrena la locomoción durante días de la semana disponibles. & & & \\
\hline \multirow{5}{*}{$\begin{array}{l}\text { Unidad Il: } \\
\text { Recorriendo mi entorno, } \\
\text { percepciones, } \\
\text { conocimiento y anhelos }\end{array}$} & 1 & Reconoce expectativas, intereses y preocupaciones. & & & \\
\hline & 2 & Reconoce espacialmente el entorno inmediato (escolar). & & & \\
\hline & 3 & Pone en práctica leyes de tránsito durante las salidas. & & & \\
\hline & 4 & Identifica puntos clave de toma de transporte público. & & & \\
\hline & 5 & $\begin{array}{l}\text { Identifica recorridos de líneas de bus específicas que transitan por } \\
\text { el sector. }\end{array}$ & & & \\
\hline \multirow{4}{*}{$\begin{array}{l}\text { Unidad III: } \\
\text { Comportamiento social } \\
\text { en el transporte público }\end{array}$} & 1 & $\begin{array}{l}\text { Identifica normas sociales requeridas para la utilización del } \\
\text { transporte público. }\end{array}$ & & & \\
\hline & 2 & Es capaz de comunicarse en beneficio de un traslado eficaz. & & & \\
\hline & 3 & $\begin{array}{l}\text { Es capaz de incorporar normas sociales como ceder el asiento, } \\
\text { limpieza en el bus, hablar en un tono de voz óptimo. }\end{array}$ & & & \\
\hline & 4 & $\begin{array}{l}\text { Realiza una rutina fluida desde que se sube al bus, deseando } \\
\text { buenos días, cancelando su pasaje, trasladándose por el pasillo, y } \\
\text { bajando en un lugar adecuado. }\end{array}$ & & & \\
\hline \multirow{3}{*}{$\begin{array}{c}\text { UNIDAD IV: } \\
\text { Situaciones imprevistas } \\
\text { y medidas de seguridad } \\
\text { en el uso del transporte } \\
\text { público }\end{array}$} & 1 & $\begin{array}{l}\text { Responde asertivamente frente a situaciones hipotéticas asociadas } \\
\text { al uso del transporte público como: tomar micro equivocada, } \\
\text { bajarse en un lugar desconocido, que el bus quede parado y } \\
\text { necesite llegar a un lugar a determinada hora. }\end{array}$ & & & \\
\hline & 2 & $\begin{array}{l}\text { Es capaz de comunicarse de forma clara su dificultad para que otro } \\
\text { le ayude. }\end{array}$ & & & \\
\hline & 3 & $\begin{array}{l}\text { Tiene claridad de las medidas de seguridad en caso de accidente } \\
\text { como: choque o incendio. }\end{array}$ & & & \\
\hline \multirow{4}{*}{$\begin{array}{l}\text { Unidad V: } \\
\text { Mis opciones de } \\
\text { locomocion y uso del } \\
\text { transporte público }\end{array}$} & 1 & Reconoce espacialmente el entorno inmediato (hogar) & & & \\
\hline & 2 & Identifica puntos clave de parada del transporte público. & & & \\
\hline & 3 & Se desplaza de forma independiente desde el hogar a la escuela. & & & \\
\hline & 4 & Identifica microbús de acuerdo con lugares de interés a recorrer & & & \\
\hline
\end{tabular}

Fuente: Elaboración propia 
racción Social -ACIS y el Cuestionario Volitivo -VQ, tanto en el contexto de la sala de clases como en la primera sesión del programa.

A manera de talleres, en el desarrollo del programa como tal, se abordan los siguientes unidades temáticas: percepción de las familias sobre el desplazamiento en comunidad; recorriendo mi entorno; percepciones, conocimiento y anhelos; comportamiento social en medios de locomoción colectiva; situaciones imprevistas y medidas de seguridad en el uso de locomoción colectiva; mis opciones de movilidad; independencia en la movilidad urbana.

Las sesiones se desarrollan con actividades grupales en aula, cada unidad inicia con un contenido teórico que se trabaja de manera didáctica, posteriormente se realizan salidas a terreno aplicando los contenidos. Adicionalmente se trabaja con la familia mediante guías en el hogar, las cuales contienen sugerencias teóricas y prácticas para apoyar el aprendizaje. Se utilizan estrategias didácticas y motivantes para fomentar el interés y potenciar la búsqueda de desafíos.

Cada unidad del Programa de Locomoción y Uso del Transporte Público se evalúa mediante la valoración funcional de los aprendizajes, mencionado previamente (tabla 2). La aprobación de cada unidad es requisito para continuar desarrollando las siguiente. Durante la última sesión del programa se aplican nuevamente la Evaluación de Habilidades de Comunicación e Interacción Social-ACIS y el Cuestionario Volitivo -VQ, comparando los resultados con los obtenidos al inicio. Se estima una duración promedio del programa de 8 meses, los cual puede variar de acuerdo al avance de cada estudiante a través de las unidades.

\section{Consideraciones éticas}

Se cuenta con el consentimiento informado escrito de las estudiantes y sus familias, el cual se aplica al inicio del programa. Este consentimiento respalda la información personal, narrativa y los dibujos elaborados por las participantes, en consecuencia, los nombres que se utilizan a continuación son ficticios.

\section{Presentación de casos}

Un equipo compuesto por terapeuta ocupacional, educadora diferencial, estudiantes y familia seleccionaron a las participantes del programa de acuerdo a sus recursos personales y ambientales, teniendo en cuenta aquellos casos en los que había interés por lograr independencia en el desplazamiento, aunque se presentaban temores frente a esta posibilidad.

Se reportan los casos de las dos estudiantes seleccionadas, de 17 y 21 años de edad y con diagnósticos de discapacidad intelectual moderada, quienes participaron en el Programa de Locomoción y Uso del Transporte Público entre los meses de marzo y noviembre de 2017. Estas jóvenes presentaban características comunes como la necesidad de lograr el uso del transporte público independiente y contar con apoyo familiar. El equipo de Terapia Ocupacional implementa el programa de forma preliminar con estas estudiantes, con miras a evidenciar su efectividad.

El objetivo general del programa fue desarrollar habilidades para la locomo- 
ción y el uso del transporte público en contextos inmediatos y mediatos, y los objetivos específicos fueron: fortalecer componentes volitivos asociados al desarrollo del programa, y desarrollar o fortalecer habilidades de comunicación e interacción social.

\section{Caso 1, Antonia}

Antonia es una joven de 21 años de edad con una discapacidad intelectual moderada, vive en San Pedro de la Paz, junto a sus padres y a una hermana de 25 años. Está escolarizada desde los 3 años, quienes la conocen la describen como una joven introvertida, pasiva, que le gusta escuchar música y utilizar las redes sociales.

El río dibujado por Antonia muestra un flujo de energía constante, no se visualiza donde nace ni donde acaba; mientras dibuja la metáfora, Antonia identifica como cualidades (ryuboyu) el hablar poco y realizar ejercicios diariamente al caminar con sus padres de la escuela a la casa y viceversa, pinta ambas maderas con color rojo y menciona que quiere resaltar esas características. Luego dibuja dos rocas (iwas) y escribe en ellas: "no me dejan salir sola" y "me aburro en mi casa"; dibuja ambas rocas con color plomo, la primera es más grande, pues la considera un gran problema. En cuanto a la paredes del río, dice: "a mis padres les cuesta dejarme hacer cosas sola" (figura 2).

Luego de este diagnóstico inicial, durante el mes de marzo se aplicó la Evaluación de Habilidades de Comunicación e Interacción Social -ACIS en la sala de clases, antes del inicio del programa, y en la primera sesión del programa. La prueba se aplicó nuevamente al finalizar el programa, en el mes de noviembre, tanto en la sala de clases como en la última sesión. Se compararon los resultados obtenidos en los dos momentos (figura 3).

Figura 2. Dibujo realizado por Antonia a partir de la metáfora del río

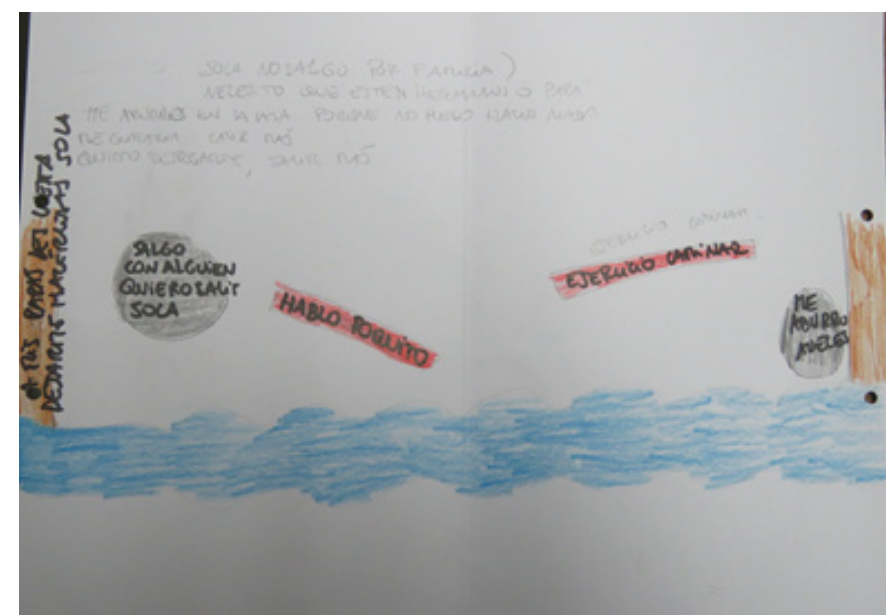

Fuente: Antonia, 25 de marzo de 2017. 
Flgura 3. Resultados obtenidos por Antonia en la Evaluación de Habilidades de Comunicación e Interacción Social -ACIS

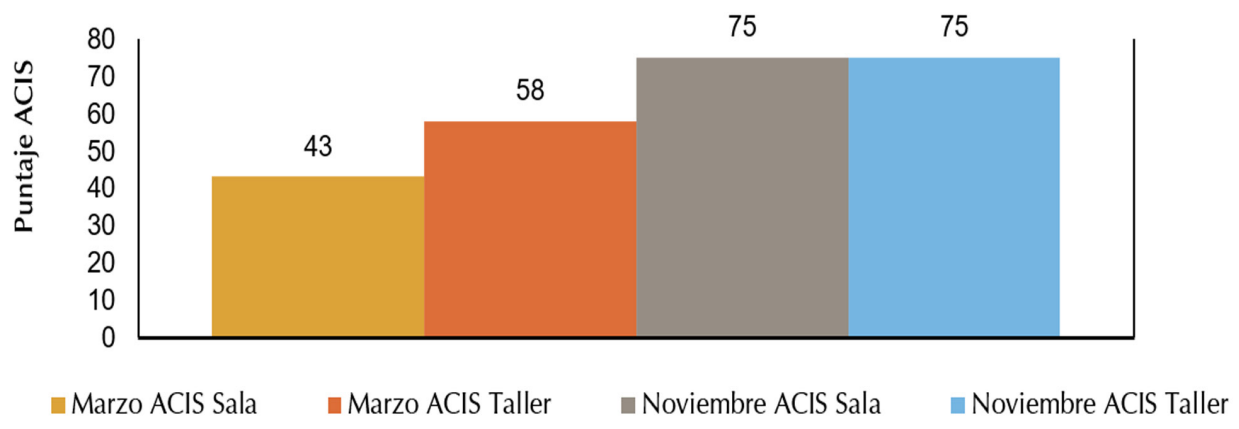

Fuente: Elaboración propia

La figura 3 muestra un aumento en las habilidades de comunicación e interacción social tanto en la sala de clases, donde pasó de 43 a 75 puntos, como durante el programa, de 58 a 75 puntos. Antonia incrementó sus habilidades en el dominio físico, pasando de un desempeño inefectivo-cuestionable a uno competente, con potencialidades al establecer contacto con otros y asumir posiciones físicas que facilitan la acción social. En el dominio de intercambio de información, las modificaciones fueron mayores, mientras en las primeras sesiones presentaba una escasa articulación del palabras, un tono de voz bajo e ininteligible, al finalizar el programa Antonia logró verbalizar sus pensamientos de forma clara; expresar peticiones, negativas y opiniones en un diálogo fluido.

De otro lado, las habilidades de Antonia al momento de iniciar interacciones alcanzaron un desempeño cuestionable, requiriendo ayuda externa para llevar a cabo demostraciones de afecto, lo que se asocia con sus características personales. En el dominio de relaciones presenta como potencialidades una conducta que favorece la comunicación con su entorno y cumplir normas sociales.

En cuanto a lo resultados del Cuestionario Volitivo - VQ aplicado al inicio y al final del programa, Antonia demostró un fortalecimiento de los componentes volitivo, con un comportamiento espontáneo, invirtiendo energía y responsabilidad adicional en las tareas y buscando desafíos permanentemente. La figura 4 muestra este aumento, pasando de 25 a 50 puntos en la sala de clases y de 28 a 49 en el programa.

Lo anterior se refleja en la transición que Antonia hizo, de usar transporte público con acompañante a la locomoción y uso del transporte público de manera independiente, ya que ha realizado esta acción en dos ocasiones durante el programa, la necesidad que siente Antonia por llevar a cabo esta tarea es relevante, por lo que sigue trabajando para asumirlo de manera permanente, realizando esporádicamente traslados de la casa a la escuela y viceversa. Se mantiene involucrada de manera autónoma, tratando de resolver conflictos y errores. 
Flgura 4. Resultados obtenidos por Antonia en el Cuestionario Volitivo -VQ

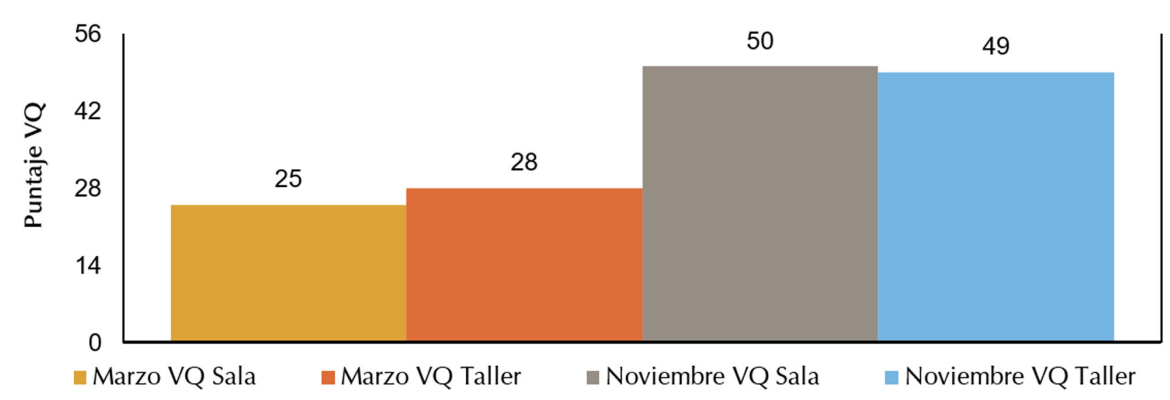

Fuente: Elaboración propia

\section{Caso 2, Tamara}

Tamara es una joven de 19 años con una discapacidad intelectual moderada, vive junto a su madre en San Pedro de la Paz. Está escolarizada desde su infancia y sus conocidos la describen como una joven extrovertida, que le gusta escuchar música, utilizar redes sociales y conversar. Dice estar interesada en aprender a usar el transporte público por si sola.
En el río dibujado por Tamara no se evidencian un inicio ni un fin aparentes, comenzó trazando una gran madera flotante (ryuboyu) y mencionando "soy porfiada"; pintó además dos rocas (iwas), una más grande y esférica con la cual señaló: "mi mamá no me deja salir sola", con la segunda, más pequeña y alargada, dijo: "suelo hablar con personas desconocidas, y a mi madre le molesta". Con su lápiz encerró todo el dibujo señalando: "el sector donde vivo es peligroso" (figura 5).

Figura 5. Dibujo realizado por Tamara a partir de la metáfora del río

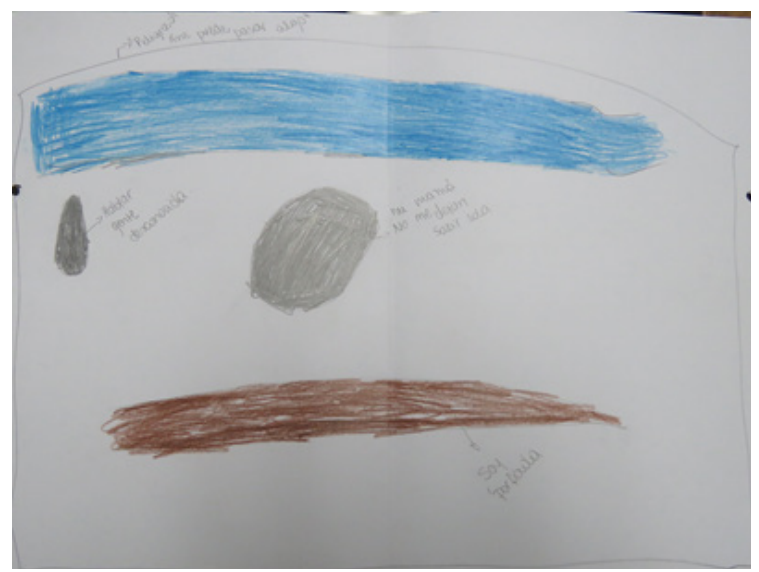

Fuente: Tamara, 25 de marzo de 2017. 
Respecto a las habilidades de comunicación e interacción social evaluadas, al final del programa se evidenciaron avances en la expresión corporal asociada a la locomoción, Tamara logró un desempeño competente en la mayoría de los ítems. Asimismo, demostró mejoría en la articulación de palabras y en la duración del discurso, sin embargo, en ocasiones utilizaba un tono de voz bajo, por lo que se deben reforzar aspectos asociados al intercambio de información.

Tamara tuvo dificultad para corregir errores, lo que se evidenció al momento de tocar el timbre del medio de transporte en un lugar equivocado, sin ser capaz de informar al chofer para que este lograra continuar su recorrido. Se observaron avances en la mayoría de los ítems de relación e intercambio de información durante el programa, desde un desempeño ineficiente a cuestionable.

La figura 6 muestra el aumento en las habilidades de comunicación e interacción social, tanto en la sala de clases, de 44 a 62 puntos, como en el programa, de 48 a 67.

En relación con los resultados del Cuestionario Volitivo- VQ, inicialmente Tamara mostraba escasa participación e iniciativa en el contexto de la sala de clases y en el programa, con un desempeño dudoso, requiriendo ser consultada para evidenciar sus aprendizajes. Sin embargo, desarrolló una motivación espontánea en la mayoría de los ítems asociados a la locomoción en actividades prácticas. Al final del programa demostró curiosidad, inicio de acciones, estuvo en capacidad de indicar objetivos y permanecer involucrada, invirtiendo energía en ello; no obstante, requería refuerzo de un tercero para intentar cosas nuevas, resolver problemas asociados al uso del transporte público en dicha tarea. Lo anterior se refleja en la figura 7, donde las puntuaciones en el componente volitivo pasan de 25 a 31 puntos en la sala de clase y de 30 a 50 en el programa. Tamara en la actualidad usa el transporte público de manera independiente en el recorrido de la casa a la escuela y viceversa.

FIgura 6. Resultados obtenidos por Tamara en la Evaluación de Habilidades de Comunicación e Interacción Social -ACIS

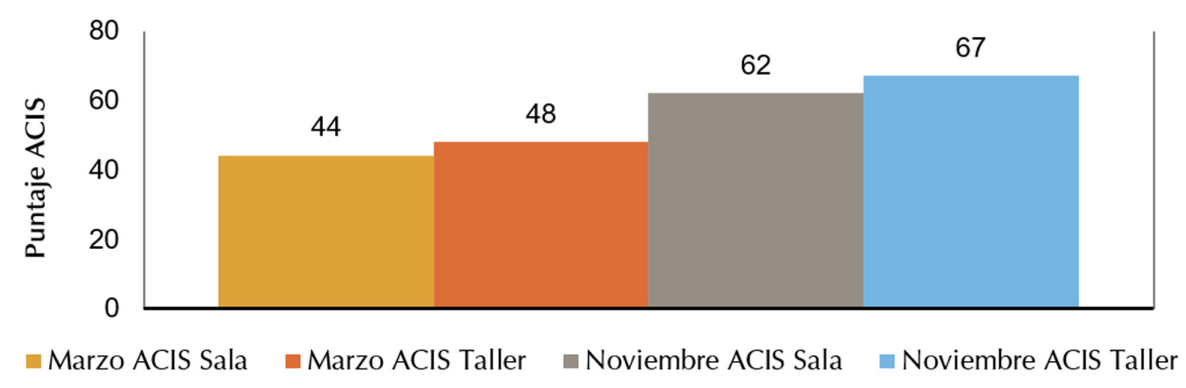

Fuente: Elaboración propia 
Flgura 7. Resultados obtenidos por Tamara en el Cuestionario Volitivo -VQ.

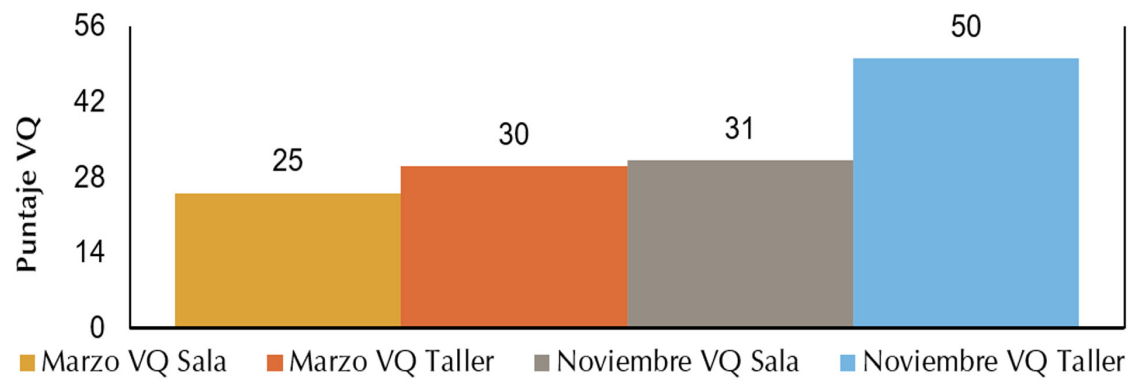

Fuente: Elaboración propia

\section{Discusión}

El Programa de Locomoción y Uso del Transporte Público plantea la relevancia de este tipo de abordaje en el sistema escolar, entendiendo que el modelo de formación que asume un establecimiento educativo incide directamente en la inclusión social de la persona con discapacidad intelectual en el futuro (Rebolledo, 2009). De este modo, la incorporación paulatina de los terapeutas ocupacionales en el contexto educativo, en este caso en Chile, ha permitido desarrollar acciones orientadas al diario vivir y abrir discusiones sobre prácticas más efectivas que contemplen las actividades cotidianas y las relaciones que se establecen en la escuela y la sociedad (Jurdi, Brunello y Honda, 2004).

Resulta enriquecedor el proceso de diagnóstico basado en el Modelo Kawa, dado que, como plantea Iwama (2008) "el modelo trata de privilegiar a la persona para que denomine y explique sus propios conceptos, en sus propias palabras y a su manera" (p.29). Por otro lado, la utilización del Modelo de
Ocupación Humana en el contexto del programa ha entregado herramientas teóricas, conceptuales y prácticas que permiten evidenciar los cambios volitivos y en las habilidades de comunicación e interacción social.

Como mencionan Haveman et al. (2013), las dificultades en el uso del transporte público y en la locomoción son temáticas constantes en la población con discapacidad intelectual; a pesar de existir evaluaciones basadas en programas de movilidad como el Listado de Evaluación de Movilidad -MAS (Kvas, Š et al., 2013), estas se basan en lo que se espera de los estudiantes en actividades de locomoción y uso del transporte público para el logro de la movilidad independiente. En su lugar, el programa que aquí se presenta está basado en los intereses, las elecciones conscientes y el fortalecimiento de procesos volitivos, demostrando, para estos dos casos, ser efectivo en el desarrollo de habilidades de comunicación e interacción, así como en la permanencia de los comportamientos que favorecen la locomoción independiente. 
De acuerdo a lo que mencionan Costabile y Brunello (2005), es fundamental comprender que programas como este, para tornarse realmente efectivos, deben hacer parte de un proceso dinámico que depende de constantes evaluaciones, reflexiones y discusiones a partir de las prácticas y vivencias. Como cualquier propuesta innovadora, esta no se hace efectiva por decreto, requiere de acciones que posibiliten su viabilidad y maduración, y de la disposición de las personas a aceptar la diversidad como condición inherente a la sociedad.

La implementación del Programa de Locomoción y Uso del Transporte Público con estas estudiantes mostró la necesidad de revisar la utilización de la Evaluación de Habilidades de Comunicación e Interacción Social -ACIS y el Cuestionario Volitivo -VQ en otros contextos donde existe mayor libertad de participación, como los recreos. Así mismo, evaluar la pertinencia de volver a utilizar la metáfora del río al finalizar el programa para analizar, desde la perspectiva de los jóvenes, cómo han variado sus apreciaciones sobre la locomoción y el uso de transporte público. Del mismo modo, es relevante realizar un seguimiento luego de la finalización del programa y fortalecer el apoyo a los padres para disminuir los miedos y preocupaciones que pudiesen perjudicar el logro de los objetivos.

Finalmente, a diferencia de los estudios de Haveman et al. (2013) y Kvas, ̌̌ et al., (2013), los cuales han probado la efectividad de algunos programas en el desarrollo de habilidades en muestras significativas de estudiantes con discapacidad intelectual, este reporte de dos casos no es representativo, de manera que se requiere de investigaciones adicionales para evaluar sus resultados. Asimismo, el poco tiempo transcurrido desde el inicio de la implementación del programa fue un factor limitante para obtener información sobre los cambios a largo plazo.

\section{Conclusiones}

Los casos de Antonia y Tamara muestran que, siendo una necesidad personal realizar elecciones ocupacionales que otorguen mayor autonomía e independencia, estas jóvenes con discapacidad intelectual pudieron desarrollar habilidades y cambios volitivos a lo largo del tiempo mediante su participación en el Programa de Locomoción y Uso del Transporte Público.

El programa permitió la exploración de habilidades, el fortalecimiento de la causalidad personal, la búsqueda de intereses, la interacción con el medio y la eliminación de factores que restringían la participación. Este programa se constituyó para las participantes en el primer paso para adquirir competencias necesarias y crear un entorno seguro para la participación comunitaria.

El enfoque propuesto da relevancia al sentir de los participantes, considerando los deseos de lograr la locomoción y el uso del transporte público como medio de liberación, descubrimiento y fortalecimiento personal. La incorporación de la visión de Terapia Ocupacional en ese tipo de programas enfatiza en reconocer a los estudiantes como gestores de sus propias transformaciones, haciéndolos conscientes de estos procesos a través de estrategias como la metáfora del río. 
Dado que el terapeuta ocupacional involucra activamente al entorno inmediato, logra conceptualizar la condición de salud de los jóvenes como un factor que no restringe su participación en ocupaciones, quebrantando así acciones excluyentes que impiden el aprendizaje. La incorporación de la familia como participante activa en el programa permite construir con los padres herramientas para brindar mayor autonomía a sus hijos y proyectar de esta manera las potencialidades hacia una conformación de identidad y competencia en la sociedad.

Las evaluaciones realizadas en la sala de clase y durante las sesiones del programa muestran que, mediante el aprendizaje significativo, los cambios percibidos se extrapolan a otros escenarios, incidiendo en la identidad personal, la competencia, y aumentando las posibilidades de adaptarse a distintos contextos de desarrollo y de llevar a cabo elecciones ocupacionales con libertad.

\section{Referencias}

Alsnih, R., \& Hensher, D. A. (2003). The mobility and accessibility of seniors in an ageing population. Transportation Research, (37), 903-916. DOI: https://doi. org/10.1016/S0965-8564(03)00073-9

Castro de Jong, D., Barrientos Lalanne, C., Castro Wolf, F., Díaz Jarufe, N., Gatti Silva, G., Guajardo Zambrano, M., Narvaéz Zavala, D., Rivera Medina, C., Torres Risso, J. \& Zúñiga Copaja, M. (2010). El Modelo Río (Kawa): cuatro estudios de caso aplicados en Chile. Revista Chilena de Terapia Ocupacional, (10), 21 - 34. DOI: https://doi. org/10.5354/0719-5346.2010.10557
Conaset \& Ministerio de Transportes y Telecomunicaciones del Gobierno de Chile. (2017). Desarrollo de objetivos de aprendizaje para los niveles de $1^{\circ}$ a $6^{\circ}$ básico en el área de educación vial. Recuperado de https://www.conaset.cl/wp-content/ uploads/2017/08/CURRÍCULUM-EDUCACIÓN-VIAL-19-05-2017_FINAL.pdf

Costabile C. \& Brunello MIB. (2005). Repercussões da inclusão escolar sobre o cotidiano de crianças com de ciência: um estudo a partir do relato das famílias. Revista de Terapia Ocupacional da Universidade de São Paulo, 16(3), 124130. DOI: http://dx.doi.org/10.11606/ issn.2238-6149.v16i3p124-130

De Las Heras, C., Geist, R., Kielhofner, G. \& Li, Y. (1998). Manual del Usuario del Cuestionario Volicional (VQ). Chicago: The Model of Human Occupation Clearinghouse, University of Illinois at Chicago.

Gobierno de Chile. (1990, 5 de mayo). Decreto 87. Aprueba planes y programas de estudio para personas con deficiencia mental.

Federación Mundial de Terapeutas Ocupacionales -WFOT. (2004). Definición de terapia ocupacional. Recuperado de http://www.wfot.org/ResourceCentre/ tabid/132/did/43/Default.aspx

Forsyth, K. (1998). Manual del Usuario de la Evaluación de las Habilidades de Comunicación e Interacción (ACIS). Chicago: The Model of Human Occupation Clearinghouse, University of Illinois at Chicago.

Haveman, M., Tillmann, V., Stöppler, R., Kvas, ̌̌. \& Monninger, D. (2013). Mobi- 
lity and public transport use abilities of children and young adults with intellectual disabilities: results from the 3-year nordhorn public transportation intervention study. Journal Of Policy \& Practice In Intellectual Disabilities, 10(4), 289-299. Doi:10.1111/jppi.12059

Heiser, X. (1995). The nature and causes of transport disability in Britain and how to overcome it. En G. Zarb (Ed.), Removing disabling barriers (pp. 123-132). London: Policy Studies Institute.

Iwama, M. \& Simó Algado, S. (2008). El Modelo Kawa (Rio). TOG (A Coruña), 5(8). Recuperado de http://www.revistatog.com/num8/pdfs/modelo2.pdf

Jurdi APS., Brunello MIB., Honda M. (2004). Terapia ocupacional e propostas de intervenção na rede pública de ensino. Revista de Terapia Ocupacional da Universidade de São Paulo, 15(1), 26-32. DOI: http://dx.doi.org/10.11606/issn.22386149.v15i1p26-32

Kielhofner, G., \& Forsyth, K. (2011). Modelo de Ocupación Humana: Teoría y aplicación. $4^{a}$ ed. Buenos Aires: Médica Panamericana.

Kvas, Š., Stöppler, R., Haveman, M., \& TiIlmann, V. (2013). Assessing mobility competences of children with intellectual disabilities: Development and results of the Mobility Assessment Schedule. Journal of Policy and Practice in Intellectual Disabilities, 10(4), 300-306. DOI: https://doi.org/10.1111/jppi.12060

Ministerio de Educación (2011). Transición para la vida adulta. Recuperado de: http://www.mineduc.cl/index2.php?id_ portal $=20$ id\&_seccion $=3007$
Quintana, M. \& Barrera, D. (2013). Guía: Educación para la Transición. Orientaciones técnico - pedagógicas y de gestión institucional para favorecer la transición hacia una vida adulta activa de estudiantes que presentan necesidades educativas especiales múltiples en el tramo comprendido entre los 14 y 26 años de edad cronológica. Santiago de Chile: Ministerio de Educación. Recuperado de http://especial.mineduc.cl/wp-content/uploads/ sites/31/2016/09/201405051722200. GUIAPARALATRANSICIONedespecial.pdf

Rebolledo, A. (2009). Configuración de la formación laboral como factor de empleabilidad en educación especial en al provincia de Ñuble, región del Bío-Bío, Chile. Horizontes Educacionales, 14 2), 21-36. Recuperado de http://www. redalyc.org/pdf/979/97915161003.pdf

Rusch, F.R., Hughes, C., Agran, M., Martin, J. E. \& Johnson, J. R. (2009). Toward self directed learning, post-high school placement, and coordinated support. Constructing new transition bridges to adult life. Career Developmental for Exceptional Individuals, 32(1), 53-59.

Schalock, R., Borthwick-Duffy, S., Bradley, V., Buntinx, W., Coulter, D., Craig, E., Gómez, S., Lachapelle, Y., Luckasson, R., Reeve, A., Shogren, K., Snell, M., Spreat, S., Tassé, M., Thompson, J., Verdugo-Alonso, M. A., Wehmeyer, M. \& Yeager, M. (2010). Intellectual disability. Definition, classification, and systems of supports. 11 Ed. Washington: AAIDD. 